\title{
Philonsorbonne
}

8 | 2014

Année 2013-2014

\section{Espace public et constellation post-nationale. Lire Strukturwandel der Öffentlichkeit à l'époque globale}

\section{Luca SCUCCIMARRA}

Traducteur : Charlotte Baby

\section{OpenEdition}

\section{Journals}

\section{Édition électronique}

URL : https://journals.openedition.org/philonsorbonne/671

DOI : 10.4000/philonsorbonne.617

ISSN : 2270-7336

\section{Éditeur}

Publications de la Sorbonne

\section{Édition imprimée}

Date de publication : 1 janvier 2014

Pagination : 177-187

ISSN : 1255-183X

\section{RÉFÉRENCE ÉLECTRONIQUE}

Luca SCUCCIMARRA, «Espace public et constellation post-nationale. Lire Strukturwandel der Öffentlichkeit à l'époque globale », Philonsorbonne [En ligne], 8 | 2014, mis en ligne le 19 janvier 2014, consulté le 10 juin 2021. URL : http://journals.openedition.org/philonsorbonne/611 ; DOI : https://doi.org/10.4000/philonsorbonne.611 


\title{
Espace public et constellation post-nationale. Lire Strukturwandel der Öffentlichkeit à l'époque globale
}

\author{
Luca SCUCCIMARRA
}

\section{1. Öffentlichkeit et ouverture}

Plus d'un demi-siècle après sa rédaction, l'œuvre de Jürgen Habermas Strukturwandel der Öffentlichkeit - dont la première édition allemande date de 1962 - continue à représenter un élément de comparaison incontournable pour quiconque veut affronter la question des dynamiques de la politique contemporaine entre État constitutionnel de droit et démocratie de masse dans une perspective théorico-critique réfléchie. Comme cela a été maintes fois souligné, les raisons de cette longévité exceptionnelle se trouvent dans la particularité même du texte : en effet, ce que nous offre Habermas dans son œuvre est bien plus qu'une simple «histoire de l'opinion publique », entendue comme phénomène socio-politique et catégorie idéologicoconceptuelle. Ce qui prend forme dans ces pages est un modèle critique et reconstructif innovant sous de nombreux aspects, une "grille de lecture » multidisciplinaire au centre de laquelle ce qui représente pour Habermas le noyau constitutif de la modernité politique à proprement parler ressort clairement : la dimension de l'Öffentlichkeit, terme difficilement traduisible, communément traduit en italien par «sfera pubblica», en anglais par «public sphere », et en français par « espace public ».

En tous cas, le choix de faire allusion à un cadre spécifique à travers un renvoi spatial qui serait implicitement contenu dans ce terme est évident et «repérable du point de vue sociologique». Dans une œuvre datant d'une dizaine d'années, le savant allemand-américain Peter Uwe Hohendahl a souligné l'exagération historique implicite dans cette option, définissant 
la notion anglaise de «public sphere " comme «a decidedly artificial term that did not exist in the English language of the $18^{\text {th }}$ and $19^{\text {th }}$ century ${ }^{1} \gg$. Plus récemment, d'autres auteurs ont contesté la pertinence même de cette traduction, soutenant qu'un terme plus adapté pour véhiculer les connotations originales du mot allemand serait «public/ness ${ }^{2} »$, ou encore «openness or openicity ${ }^{3}$ ». Mon but n'est pas ici de m'attarder sur de complexes controverses terminologiques. Cependant, me semble intéressante, d'un point de vue théorique et historique, l'allusion - issue sans aucun doute de la pensée de Arendt - à la dimension de l'ouverture, que de nombreux interprètes de l'Öffentlichkeit de Habermas ont mise au centre de leur réflexion, même d'un point de vue linguistique. À ce niveau du discours, cette conception normative de l'Öffentlichkeit entre clairement en jeu comme champ d'interaction discursive caractéristique; ce dernier est caractérisé, dans toutes ses variantes, par des principes constitutifs communs qui représentent un des aspects les plus particuliers et influents de la réflexion du jeune Habermas. C'est une approche développée dans l'essai de 1962, en particulier dans le long excursus sur les institutions de l'espace public, qui reste un des passages les plus connus de cette œuvre: c'est dans ces pages, en effet, que l'Öffentlichkeit apparaît comme un modèle idéal d'interaction communicative à même de garantir une détermination consensuelle du bien commun qui ne soit déformée par aucun intérêt partisan; et ce en vertu de ses propres «critères institutionnels » fondamentaux : l'abstraction systématique des inégalités de status, l'absence de limite au processus de problématisation réfléchie des questions d'intérêt commun, et surtout le refus de toute forme de fermeture subjective «vers l'extérieur ».

Néanmoins, on ne doit pas se laisser leurrer par l'universalité prétendue de cet idéal d'ouverture intersubjective. Dans la configuration historicocritique bien particulière que Habermas attribue à sa réflexion, ce champ discursif est aussi un espace structuré de relations sociales, caractérisé tout comme l'auteur ne manque de le souligner - par des hypothèses matérielles de constitution précises. De ce point de vue, s'il est vrai que le paradigme de l'Öffentlichkeit, au coeur de la reconstruction de Habermas, puise ses origines dans ce modèle universaliste d' « espace public littéraire » centré sur l'idéal d'une confrontation entre les «gens de lettres » au nom du principe du « simplement humain », il est tout aussi vrai que l'histoire de ce que Habermas lui-même définit comme «politische Öffentlichkeit (espace

1. P. U. Hohendahl (éd.), Öffentlichkeit - Geschichte eines kritischen Begriffs, Stuttgart, J. B. Metzler, 2000, p. 1.

2. L. Splichal, Public Opinion: Developments and Controversies in the Twentieth Century, Rowman \& Littlefield, 1999.

3. H. Kleinsteuber, Habermas and the Public Sphere: From a German to a European Perspective, disponible à l'adresse suivante :

http://www.razonypalabra.org.mx/anteriores/n55/hkleinstuber.html.

4. J. Habermas, Strukturwandel der Öffentlichkeit. Untersuchungen zu einer Kategorie der bürgerlichen Gesellschaft, Francfort, Suhrkamp, [1962] 1990, p. 97 et suivantes. 
public politique) » est une vicissitude fermement inscrite dans la dynamique évolutive de l'État territorial souverain moderne. Selon l'interprétation de l'auteur, elle est fondée sur le processus de transformation historico-sociale qui, du contexte indifférencié d'action typique du monde féodal, a mené à l'émergence des divers domaines du pouvoir public et de l'œuvre économique, et, par suite, à la genèse de ce «domaine de la circulation des marchandises et du travail social», qui, dans le lexique de la pensée moderne, acquerra le nom de société civile : un contexte d'action "privé », mais soumis à « une surveillance et une gestion publique », et donc perçu par les particuliers eux-mêmes comme un domaine d'intérêt général important pour tous.

C'est justement à partir de ces deux passages évolutifs fondamentaux, dans lesquels l'histoire du caractère étatique moderne s'entremêle avec celle de l'économie de marché capitaliste naissante, mais aussi avec la genèse d'un nouveau système de circulation de l'information fondé sur la presse périodique et sur les nouveaux lieux de rencontre de la socialité bourgeoise (cafés, clubs, salons), que l'ensemble des «particuliers » peut dépasser sa propre dislocation spatio-temporelle pour se fondre en un sujet collectif de nouveau genre, le publicum, destiné à s'imposer progressivement comme «contrepartie abstraite du pouvoir public, consciente de soi-même comme propre interlocuteur ${ }^{5} »$. Sur cette base, l' « espace public bourgeois » peut donc être défini comme l'espace d'action des particuliers qui, réunis en public, revendiquent, contre ce même pouvoir étatique, le droit de trouver un accord avec ce dernier sur les règles générales du commerce dans le cadre de l'« échange de marchandises et du travail social ». C'est-à-dire une sorte de tribunal où le pouvoir public est appelé à rendre compte de ses propres actions - et donc à se légitimer - face à une nouvelle instance critique ; une sorte de noyau de constitution de la publicité bourgeoise : l'opinion publique.

Dans le développement de son traité, Habermas s'attarde en particulier sur le processus diversifié d'institutionnalisation de cet «espace public à fonctions publiques » depuis sa première apparition dans l'Angleterre de la Glorious Revolution jusqu'à la consécration définitive de ce changement au XIX ${ }^{\mathrm{e}}$ siècle avec l' «État de droit bourgeois sous forme de gouvernement parlementaire » : dans un tel modèle constitutionnel, cette dimension obtient « le statut normatif d'un organe d'auto-médiation de la société civile avec un pouvoir étatique répondant à ses exigences », et s'impose ainsi comme le principe même d'organisation de l'État. Mais il insiste aussi sur la valence critique et normative intacte que le paradigme de l' «Öffentlichkeit », s'il est valorisé de manière adéquate, peut acquérir même dans les conditions politiques dégradées de la démocratie de masse de la fin du $\mathrm{XX}^{\mathrm{e}}$ siècle. Ainsi soumise à l'approche dialectique d'une critique de l'idéologie à caractère francfortois, la constellation idéale incarnée dans le revers politicoconstitutionnel de l'«espace public bourgeois» dévoile une dimension 
universaliste de grande valeur qui dépasse les limites de son propre conditionnement historique pour promettre «une totale libération de la réalité présente sans fuite dans l'au-delà » ${ }^{6}$ : elle se révèle coöncidente avec l'utopie d'une complète transparence du politique, un espace de visibilité absolue des processus de gouvernement qui, en liant toute l'activité étatique au consensus libre et discursivement arbitré de la population entière, «tend déjà à une élimination de l'État comme instrument de domination en général ${ }^{7}$.

Dans l'organisation dialectique et critique de Strukturwandel der Öffentlichkeit, cette utopie de rationalisation communicative de la domination, loin de représenter une abstraction qui doit être libérée de la matérialité des processus sociaux, demeure incarnée dans le sens objectif des formes constitutionnelles de l'État de droit parlementaire, jusque dans les conditions changeantes de la démocratie de masse ; et cette «transcendance dans l'immanence» est le moment de vérité qui place la conception bourgeoise de l'espace public au-dessus de sa propre fonction idéologique de dissimulation d'une domination de classe indéniable. Pour cela, transmettre le sentiment de sa validité universelle représente, selon Habermas, le principal objectif d'une théorie critique de la société, même là où tout semble confirmer le triomphe d'une utilisation purement manipulatrice de l'espace public politique. Ainsi, l'«Öffentlichkeit» cesse d'être simplement une catégorie historico-sociologique d'analyse pour se transformer en une instance philosophique et en une prospective politique pour le présent ${ }^{8}$.

\section{Habermas et la critique}

À partir du début des années 1960, l'élévation du paradigme habermassien de l'«Öffentlichkeit» en principe général de légitimité politico-constitutionnelle des systèmes démocratiques représentatifs a suscité de nombreuses critiques. Dans une contribution récente sur ce thème, Nancy Fraser a distingué une première ligne de débat qui limite son enquête à «the legitimacy of public opinion along lines beyond those pursued by Habermas ${ }^{9}$ " et une deuxième "stream of criticism», qui a radicalisé «Habermas's problematization of the efficacy of public opinion», analysant, dans une prospective systémique, les «structural forces»le pouvoir économique privé et les intérêts bureaucratiques qui s'y

6. Ibid., p. 112.

7. Ibid., p. 152.

8. Pour une analyse plus soignée de cette dimension de la conception de Habermas de l'«Öffentlichkeit», voir L. Scuccimarra, «La trasparenza del politico. Habermas e il paradigma della "sfera pubblica" », in Giornale di storia costituzionale, n 6, 2003, p. 35-59.

9. N. Fraser, «Transnationalizing the Public Sphere», dans Theory, culture and society, $\mathrm{n}^{\circ} 24 / 3,2007$, p. 11. 
entrelacent - «that block the flow of communicative power from civil society to the state», privant ainsi «discursively generated public opinion of political muscle ${ }^{10} »$.

La notoire objection de classe est l'exemple le plus ancien du premier genre de critique. Habermas a été accusé de toutes parts d'avoir idéalisé la forme de vie de la bourgeoisie, adhérant ainsi in toto à une représentation sociale mystifiante, elle-même expression de la domination de classe. Le choix de concentrer l'attention sur l' "espace public bourgeois» pleinement exprimé dans la préface du volume - est l'objet d'une contestation particulière, puisque, selon cette interprétation, cet «espace » est identifié à tort comme la matrice historique d'instances émancipatoires à portée universelle $^{11}$. Face à cette critique des conditionnements de classe, il faut aussi rappeler l'objection de genre pour son importance globale, c'est-àdire la critique des fondements «sexués » de l' « espace public bourgeois », avancée plusieurs fois par la pensée féministe. Outre les prémisses philosophiques du modèle, ce sont en effet ses propres fondements structuraux d'opérativité qui sont remis en question dans ce cas, en commençant par cette dichotomie public/privé dans laquelle de nombreux représentants des gender studies ont vu une personnification illégitime des rôles sociaux traditionnels ${ }^{12}$.

Il s'agit là d'une approche qui, au-delà de toute autre considération, représente une première tentative de problématiser la structure spatiale rigide qui, contre tout principe d'ouverture intersubjective, caractérise le paradigme de Habermas depuis son origine, conditionnant ainsi en profondeur les mêmes applications critiques et normatives. Nancy Fraser a souligné qu'aucun des protagonistes de cette approche déconstructive de l'«Öffentlichkeit»-ni aucun représentant des autres lignes de critique consolidées à partir de ce modèle - n'a propulsé la portée de son propre geste théorique suffisamment loin pour mettre réellement en question le «Westphalian framing of political space » qui, depuis toujours, caractérise et conditionne le modèle de revitalisation communicative de l'espace public politique élaboré par le jeune Habermas. Bien au contraire, «still oriented to the prospects for deliberative democracy in a bounded political community », tous ces auteurs, en dépit de leurs points de discorde plus ou moins radicaux, ont continue «to identify the public with the citizenry of a territorial state », faisant leur les deux autres prémisses structurales du modèle de Habermas: "the assumption of a national economy, whose proper steering by the democratic state remained a principal topos of

10. Ibid., p. 12.

11. P. Hohendahl, «Critical Theory, Public Sphere and Culture : Jürgen Habermas and his Critics », New German Critique, n 6, 1979, p. 89-118 ; J.-V. Koivisto, «Das Comeback kritischer Theorien der Öffentlichkeit », Das Argument, 201, 1993, p. 722 et suivantes.

12. Sur les limites du rapport public-privé classique dans une optique féministe : S. Benhabib, «Models of Public Space: Hannah Arendt, the Liberal Tradition and Jürgen Habermas », in C. Calhoun (éd.), Habermas and the Public Sphere, Massachusetts/Londres, MIT Press, 1992, p. 89 et suivantes. 
public sphere debate» et la référence à ce dernier comme à un processus communicatif territorialement délimité, «conducted in the national language through the national media». Animés par le même projet politique que le jeune Habermas, ils apparaissent donc engagés, dans une dernière analyse, «to further deliberative democracy in the modern territorial state ${ }^{13} »$.

S'agit-il vraiment du moyen le plus adapté pour questionner de nouveau aujourd'hui le nœud crucial des fonctions politiques de l'espace public, situé au centre de la grande structure théorique et historique de Strukturwandel der Öffentlichkeit? Répondre de manière affirmative signifierait vouloir ignorer le contexte phénoménologique bien plus problématique auquel, plus de cinquante ans après la publication de cette œuvre, les systèmes démocratiques et représentatifs contemporains sont appelés à se confronter ; en particulier, les trois facteurs de crise cruciaux qui, selon un diagnostic plutôt partagé, semblent actuellement porter atteinte à cette même capacité d'autogouvernement de la société à capitalisme avancé : la crise de souveraineté produite par les processus de globalisation et par la genèse des lieux extra-étatiques et semi-étatiques de décisions politiques ${ }^{14}$; la crise de complexité induite par les processus de différenciation sociale au contrôle et à la gestion toujours plus difficiles ${ }^{15}$; et la crise de discursivité produite par la multiplication des flux de communication médiatisée et par la fragmentation et la spécialisation des espaces de confrontation $\operatorname{argumentative~}^{16}$. Ce qui émerge de l'enchevêtrement et de la superposition de ces dynamiques de crise diversifiées est un scénario évolutif complexe qui semble mettre radicalement en question ces mêmes prémisses spatiotemporelles du modèle habermassien de l'Öffentlichkeit, s'il est vrai comme cela a été soutenu - que, d'un point de vue intrinsèquement fonctionnel au moins, les flux politico-communicatifs opérant dans les sociétés globalisées et médiatisées de notre époque sont «de plus en plus transnationaux ou post-nationaux en référence à chacun des éléments constitutifs de l'opinion publique ${ }^{17}$ ».

\section{Penser l'espace public aujourd'hui}

Tout d'abord, il n'est pas possible d'aborder cet aspect décisif de la question sans tenir compte des profonds changements qui sont entrés en jeu

13. N. Fraser, Transnationalizing the Public Sphere, op. cit., p. 13.

14. U. Beck, «Wie wird Demokratie im Zeitalter der Globalisierung möglich?», in U. Beck (éd.) Politik der Globalisierung, Francfort, Suhrkamp, 1998.

15. D. Zolo, Il principato democratico. Per una teoria realistica della democrazia, Milan, Feltrinelli, 1992, p. 73 et suivantes; U. Beck, Risikogesellschaft: auf den weg in eine andere Moderne, Francfort, Suhrkamp, 1986.

16. W. Privitera, Sfera pubblica e democratizzazione, Rome-Bari, Laterza, 2001, p. 152 et suivantes ; D. Innerarity, El nuevo espacio público, Madrid, Espasa Calpe, 2006.

17. N. Fraser, Transnationalizing the Public Sphere, op. cit., p. 19. 
dans la conception de Habermas de l'Öffentlichkeit ces dernières années. $\mathrm{Au}$ cours de son riche parcours intellectuel, Habermas s'est en effet détaché progressivement de la constellation thématique explorée dans l'essai de 1962, pour donner vie au plus ample et au plus ambitieux programme de refondation des sciences sociales que la pensée contemporaine connaisse, bien qu'en totale continuité avec l'approche critique et émancipatoire caractéristique de ses premiers écrits. Dans la préface de la deuxième édition de Strukturwandel der Öffentlichkeit, il a voulu répondre lui-même des principaux changements qui se sont opérés par rapport à son cadre théorique d'origine : il a indiqué les passages-clés d'un tel développement dans a) l'adoption d'une approche pragmatique et linguistique - la théorie de l'acte communicatif - en vue de "mettre en exergue un potentiel rationnel inné dans la propre pratique communicative quotidienne» et b) dans la substitution de la conception «totalitaire» de la société et de l'auto-organisation sociale caractéristique de ses premiers écrits par une modalité représentative plus articulée, fondée sur la distinction entre champs d'action intégrés en termes d'acte communicatif - proches de la catégorie de «monde vital»- et domaines systémiques d'action comme l'économie et l'apparat administratif de l'État, intégrés au contraire sur la base de moyens non linguistiques -1 'argent et le pouvoir ${ }^{18}$.

Il suffit de lire le huitième paragraphe de l'œuvre Faktizität und Geltung, qui est au jour d'aujourd'hui la dernière contribution systématique de Habermas sur le front de la théorie politique et sociale, pour toucher du doigt les effets produits par ce double changement de paradigme sur sa conception originelle de l'Öffentlichkeit comme espace de constitution discursive d'une volonté commune rationnelle libre. Si dans l'essai de 1962, l'objet de l'analyse était un «espace public » au singulier comme espace omni-compréhensif d'interaction discursive, c'est une multiplicité de contextes communicatifs qui est explorée et interrogée, irréductible à un champ unifié et indifférencié. Dans cette optique, le pivot de la dynamique communicative est toujours un public de publics, différenciés sur une base spatiale, temporelle, fonctionnelle ou thématique. Une image réticulée significative du niveau de fragmentation qui, dans les sociétés complexes, caractérise la communication politique, chevauche ainsi la métaphore sphérique utilisée dans le passé. La formation de l'opinion, écrit Habermas à ce propos,

s'effectue dans le cadre d'un réseau ouvert et inclusif d'espaces publics subculturels qui se recoupent et dont les frontières temporelles, sociales et matérielles sont mouvantes. À l'intérieur d'un cadre garanti par la Constitution, les structures d'un tel espace public se développent d'une façon plus ou moins spontanée. Les flux communicationnels en principe illimités passent par les espaces publics formés à l'intérieur des associations qui constituent des

18. J. Habermas, «Vorwort zur Neuauflage $1990 »$, in Strukturwandel der Öffentlichkeit, p. 33 et suivantes. Mais sur les fondements gnoséologiques de ces passages, voir J. Habermas, Theorie des kommunikativen Handelns, Francfort, Suhrkamp, 1981. 
éléments informels de l'espace public général. Dans leur totalité, ils constituent un ensemble « sauvage » qui se dérobe à toute organisation globale ${ }^{19}$.

C'est justement dans l'optique de cette relecture pluraliste et « anarchiste» de la dynamique de l'espace public que la reconstruction de Habermas s'est enrichie de nouvelles lignes d'approfondissement thématique, capables de conférer une forme concrète majeure à la représentation de la démocratie de masse : je me réfère en particulier à la réflexion sur le phénomène crucial des mouvements sociaux, totalement absent dans le modèle reconstructif de Strukturwandel der Öffentlichkeit, et identifié à présent comme l'expression la plus typique de la capacité de production spontanée de l'opinion caractéristique du «public des citoyens $»^{20}$. En outre, au centre de l'analyse de Faktizität und Geltung, apparaissent des dynamiques très lointaines du topos d'un processus d' «auto-éclaircissement collectif d'individus intéressés au bien commun»: on enquête sur la dynamique d' «auto-identification » (et «autolégitimation») qui caractérise tout phénomène d'agrégation dans le «public des citoyens », mais on enquête aussi sur les actes de désobéissance civile vus comme « dernier moyen pour procurer une attention particulière et une influence de service public et politique sur les arguments d'opposition », et on enquête même sur le risque d'un conflit intersubjectif radical qui va toujours de pair avec le développement d'une communication publique authentique. Conformément au «virage éthique » qui caractérise la dernière période de la théorie de Habermas, l'objet de confrontation discursive dans l'espace public n'est plus seulement la composition des intérêts partisans, dans une interprétation socialement partagée du bien commun, mais bien plus une lutte radicale pour la reconnaissance des besoins et des identités, qui cite à comparaître la manière même d'être des sujets individuels et collectifs, ouvrant ainsi l'espace public à une perspective expérimentale « riche de conflits et de "formes" de vie génératrices de sens » ${ }^{21}$.

Il est évident ici que la conception de Habermas de l'espace public s'ouvre au monde variable de l'expérience des sociétés capitalistes tardives, à la dynamique de globalisation et au processus d'intersection culturelle qui en représente un des aboutissements les plus visibles. Tout comme et à vrai dire bien plus que la théorie de la justice de Rawls, la politique délibérative de Habermas semble considérer le «fait du pluralisme » comme le point de départ de la construction discursive d'une véritable «solidarité entre étrangers ». Cela suffit-il pour dire que nous sommes en présence d'un changement de réflexion radical concernant les fondements constitutifs de l'Öffentlichkeit comme espace structuré de rapports sociaux ? Il semble que non s'il est vrai que, dans cette version, le modèle de Habermas reste aussi

19. J. Habermas, Faktizität un Geltung. Beitrage zur Diskurstheorie des Rechts und des demokratischen Rechtsstaats, Frankfurt a. M., Suhrkamp, 1992 (trad. fr. : Droit et démocratie, Paris, Gallimard, 1997, p. 333).

20. Ibid., p. 438.

21. Ibid., p. 365. 
fondé sur une séparation rigide entre espace public et espace privé qui se précise grâce au recours à la catégorie subjective du monde vital $l^{22}$, et que malgré la prise de distance ostentatoire par rapport aux tournures de style de la critique francfortoise de l'industrie culturelle - le rôle que Habermas est disposé à reconnaître aux nouveaux médias dans le processus de constitution d'un espace public politique efficace demeure peu clair. Partant de ce point de vue, l'impression qui ressort de la lecture de la prose non linéaire de Faktizität und Geltung est en effet qu'il continue à considérer la « discussion critique » - c'est-à-dire la confrontation réfléchie entre les divers argumentations, opinions et points de vue au sein d'un lieu commun comme l'instrument principal de constitution d'une véritable publicité politique. C'est un argument qui semble confirmer à plusieurs niveaux d'analyse l'incapacité du philosophe allemand de s'affranchir du barycentre radicalement national et étatique du discours caractérisé de Strukturwandel der Öffentlichkeit.

À présent, il n'y a plus aucun doute: une discussion adéquate sur cet aspect spécifique de la conception plus récente de Habermas de l'Öffentlichkeit requerrait une confrontation explicite avec la ligne de réflexion «post-nationale » qu'il a inaugurée dès la moitié des années 1990 en réponse aux défis cruciaux de l'«époque globale». Tout cela nécessiterait en particulier un examen approfondi des nombreuses contributions consacrées aux dilemmes fondateurs d'une démocratie européenne, en considérant que la réponse de Habermas aux représentants tétragones de la no demos thesis a assumé dès le début la forme ambitieuse d'une refondation communicative du vocabulaire «constituant» moderne, justement centrée sur le couple catégorique société civile - espace public ${ }^{23}$. Je me limiterai ici à souligner les résultats constructifs et normatifs insatisfaisants qui sont produits, même à ce niveau du discours, par une forme de monisme délibératif manifestement tracé sur l'idéal-type de la citoyenneté démocratique dans la version fermée et territorialement délimitée propre à l'expérience des États nationaux : comme pour dire que, malgré tous les efforts de renouveau de sa structure catégorique, Habermas a su opposer aux instances « républicaines dépassées » des représentants du scepticisme étatique européen l'idéal «néo-républicain» d'une "société européenne des citoyens », fondée précédemment sur la reproduction à grande échelle des mêmes dispositifs fondateurs opérationnels au niveau national $^{24}$. C'est une approche qui, au-delà de ces évidentes limites théoriques, apparaît actuellement destinée à se diriger vers des difficultés applicatives insurmontables, dans la mesure où «l'interdépendance pragmatique » entre principe national et idée démocratique a démontré une

22. R. Heming, Öffentlichkeit, op. cit., p. 234 et suivantes.

23. J. Habermas, « Nazione, Stato di diritto, Democrazia», in F. Cerutti (éd.), Identità $e$ politica, Roma-Bari, Laterza, 1996, p. 210 et suivantes.

24. J. Habermas, Die postnationale Konstellation und die Zukunft der Demokratie, in Id., Die postnationale Konstellation. Politische Essays, Francfort., Suhrkamp, 1998. 
capacité de résistance décidément supérieure à ce que pouvaient imaginer les défenseurs d'une approche « post-nationale ${ }^{25}$.

$* * *$

Personnellement, je suis enclin à croire que le dernier mot du philosophe allemand sur le rapport entre «espace public» et «condition postnationale » est celui exprimé il y a une dizaine d'années dans l'essai Hat die Konstitutionalisierung des Völkerrechts noch eine Chance? : il s'agit d'une prise de position en faveur d'une forme de "constitutionalization of international law», ancrée dans " the kinds of democratic processes of opinion- and will-formation that can only be fully institutionalized within constitutional states ». Habermas écrit :

Only within states does the organizational part of the constitution secure citizens equal access to the politically binding decisions of the government through institutionalized publics, elections, parliaments, and other forms of participation. Only within constitutional states do administrative mechanisms exist to insure the equal inclusion of citizens in the legislative process. Where these are lacking, as in the case of the constitutions of international organizations, there is always the danger that the "dominant" interests will impose themselves in a hegemonic manner under the guise of impartial laws ${ }^{26}$.

Cela étant dit, il n'est pas certain que la vicissitude évolutive du paradigme de Habermas de l'Öffentlichkeit doive se conclure de cette manière. Au contraire, au cours des dernières années, plusieurs auteurs ont proposé une relecture de ce paradigme, centrée justement sur le fait de surmonter la conception moniste du rapport espace public - citoyenneté. Cette relecture semble être une réponse aux perplexités suscitées par le revirement «identitaire» de Habermas ${ }^{27}$. Seyla Benhabib nous offre une contribution importante dans cette perspective, et cela à travers son modèle de fédéralisme républicain. Dans une telle configuration, parmi les effets les plus notoires du système de processus d'intégration juridico-politique de l'U.E., il faut dénombrer trois composants, indépendants les uns des autres, de la « désagrégation » progressive du modèle unitaire de citoyenneté traditionnel: identité collective, privilège de l'appartenance politique et revendications sociales $^{28}$. Au lieu de continuer à laisser guider notre

25. J. Lacroix, Une citoyenneté européenne est-elle possible?, disponible à l'adresse suivante : www.laviedesidees.fr.

26. J. Habermas, «Hat die Konstitutionalisierung des Völkerrechts noch eine Chance? », in Id., Der gespaltene Westen, Francfort, Suhrkamp, 2004 (trad. angl. : «Does the Constitutionalization of International Law Still Have a Chance ? , in J. Habermas, The Divided West, Malden, Polity, 2006, p. 141 et suivantes).

27. J. Lacroix, «Does Europe Need Common Values? Habermas vs Habermas », European Journal of Political Theory, $\mathrm{n}^{\circ}$ 8, 2009, p. 141 et suivantes.

28. S. Benhabib, The Rights of Others. Aliens, Residents and Citizens, Cambridge, Cambridge University Press, 2004, p. 129 et suivantes. 
imagination politique par un modèle d'autodétermination désormais obsolète, nous devrions plutôt nous forcer à imaginer « des formes d'action et de subjectivité politique capables d'avancer de nouvelles modalités de citoyenneté politique ${ }^{29} »$.

Dans le débat actuel se cachent d'autres concepts : la conception de la demoi-cracy - une forme de démocratie transnationale centrée sur la reconnaissance d'une "pluralité d'institutions et de communauté » bien plus ample et variée - imaginée par James Bohman en référence à l'atelier constitutionnel potentiel de l'Union Européenne ${ }^{30}$; la réflexion stimulante sur «l'opinion publique mondiale » développée par Halke Brunkhorst à partir des dynamiques complexes de la politique internationale actuelle ${ }^{31}$; et enfin, il y a la politics of framing, autrement dit la confrontation radicale avec les mêmes hypothèses de constitution de notre discours sur la politique, promue par Nancy Fraser de manière incessante dans la dernière période de son parcours intellectuel : l'élaboration d'une nouvelle théorie de l'espace public « post-Habermas » fait partie intégrante de cette approche, capable de répondre aux grands dilemmes fondateurs produits par les dynamiques politiques et juridiques de l'«époque globale». Selon la philosophe américaine, le fait de se confronter d'un point de vue fondateur et normatif à de telles questions signifie en outre offrir une contribution décisive pour l'élaboration d'une véritable "théorie critique pour un monde postwestphalien ». Pour arriver à cet objectif, il ne suffit pas que les experts des études culturelles et médiologues fassent une carte des flux communicatifs existants. Il faut, bien au contraire, que les représentants d'une théorie critique de la politique et de la société repensent complètement «the theory's core premises concerning the legitimacy and efficacy of public opinion ». C'est uniquement de cette manière que la réflexion sur de nouveaux espaces de la politique pourra se considérer vraiment à la hauteur des intentions originelles de Habermas, qui étaient de contribuer à la lutte pour l'émancipation politique et sociale dans le contexte polarisé des démocraties représentatives contemporaines ${ }^{32}$.

(Traduit de l'italien par Charlotte Baby)

29. Ibid., p. 174 et suivantes.

30. J. Bohman, Democracy across Borders. From Dêmos to Dêmoi, Cambridge-Londres, MIT Press, 2007.

31. H. Brunkhorst, Solidarität : Von der Bürgerfreundschaft zur globalen Rechtsgenssenschaft, Francfort, Suhrkamp, 2002.

32. N. Fraser, Transnationalizing the Public Sphere, op. cit., p. 24. 